\title{
Melanocytic Nevus on the Rectal Mucosa Removed Using Endoscopic Submucosal Dissection
}

\author{
Seong Min Kim, Yoon Ji Shin, Ju Sung Sim, Beon Jae Lee, Moon Kyung Joo, Jong-Jae Park and Young-Tae Bak \\ Division of Gastroenterology, Department of Internal Medicine, Korea University Guro Hospital, Korea University College of Medicine, Seoul,
} Korea

Melanocytic nevus is the benign proliferation of melanocytes. The most common location of melanocytic nevus is the skin of the extremities; however, there are few case reports of melanocytic nevus at the rectal mucosa. No prior case of malignant melanoma from melanocytic nevus at the rectal mucosa has been reported; therefore, it is unclear whether resection should be performed or close observation is sufficient. However, the potential malignant transformation of melanocytic nevus should be considered, including melanocytic nevus on the rectum. Melanocytic nevus of the skin can be removed by surgical excision; however, due to rare incidence on the mucosa of the gastrointestinal tract, the optimal treatment for rectal melanocytic nevus remains controversial. Here, we report the first case of melanocytic nevus on the rectal mucosa that was removed by endoscopic submucosal dissection. This case report provides useful information about the optimal management of rectal melanocytic nevus. Clin Endosc 2016;49:391-394

Key Words: Nevus, pigmented; Melanoma; Endoscopic submucosal dissection

\section{INTRODUCTION}

The annual occurrence of malignant melanoma has been dramatically increasing over the past few decades, and the exposure to ultraviolet light is assumed to be a major risk factor. ${ }^{1}$ Other risk factors including the high number of typical melanocytic nevi, presence of atypical nevi, personal history of melanoma, and several genetic factors including increased frequency of CDKN2A mutations have been reported..$^{2-4}$ Additionally, familial atypical multiple mole and melanoma syndrome are reportedly related with a higher incidence of malignant melanoma. ${ }^{5}$

Typical melanocytic nevi occur at photo-exposed areas, where the risk of cutaneous melanoma greatly increases, and

Received: October 5, 2015 Accepted: October 26, 2015

Correspondence: Jong-Jae Park

Division of Gastroenterology, Department of Internal Medicine, Korea University Guro Hospital, Korea University College of Medicine, 148 Gurodong-ro, Gurogu, Seoul 08308, Korea

Tel: +82-2-2626-1027, Fax: +82-2-2626-1019, E-mail: gi7pjj@korea.ac.kr

(cc) This is an Open Access article distributed under the terms of the Creative Commons Attribution Non-Commercial License (http://creativecommons.org/ licenses/by-nc/3.0) which permits unrestricted non-commercial use, distribution, and reproduction in any medium, provided the original work is properly cited. they are generally round with a uniform color and a diameter of $5 \mathrm{~mm}$ or less. ${ }^{6}$ Melanocytic nevi are infrequently pigmented to the mucosa, and several cases of melanocytic nevus at the rectal mucosa have been reported. ${ }^{2-4}$ However, there are no prior reports of the malignant transformation of rectal melanocytic nevus or its treatment. Like melanocytic nevus of the skin, rectal melanocytic nevus has the potential to transform to malignant melanoma. Thus, endoscopic resection of rectal melanocytic nevus might be an alternative treatment option. Here, we report the first detailed case report of rectal melanocytic nevi resected using endoscopic submucosal dissection (ESD).

\section{CASE REPORT}

A 70-year-old male without a medical history of remarkable illness visited our hospital for screening colonoscopy. The patient had no previous experience of colonoscopy and successfully underwent propofol-based conscious sedation. During the procedure, a $1.0 \times 0.8 \mathrm{~cm}$ hyperpigmented and slightly elevated lesion was observed at the far distal rectum near from dentate line (Fig. 1). A tiny piece of tissue was 
taken from the lesion, and the acquired specimen was tested under H\&E staining and immunohistochemical staining for tumor proliferation using the Ki-67 labeling index. The Ki67 labeling index was less than $1 \%$, and the results of the hematoxylin-eosin-stain identified it as a melanocytic nevus (Fig. 2). Although there have been no previous written reports of the transformation of melanocytic nevus at the rectum to malignant melanoma, a large melanocytic nevus might have the potential for malignant transformation. The patient was recommended periodic colonoscopy or endoscopic resection, and he decided to receive therapeutic endoscopic resection after 10 months of colonoscopic examinations.

The hyperpigmented lesion at the rectum showed no tion-based sedation, standard ESD was performed (Fig. 3).

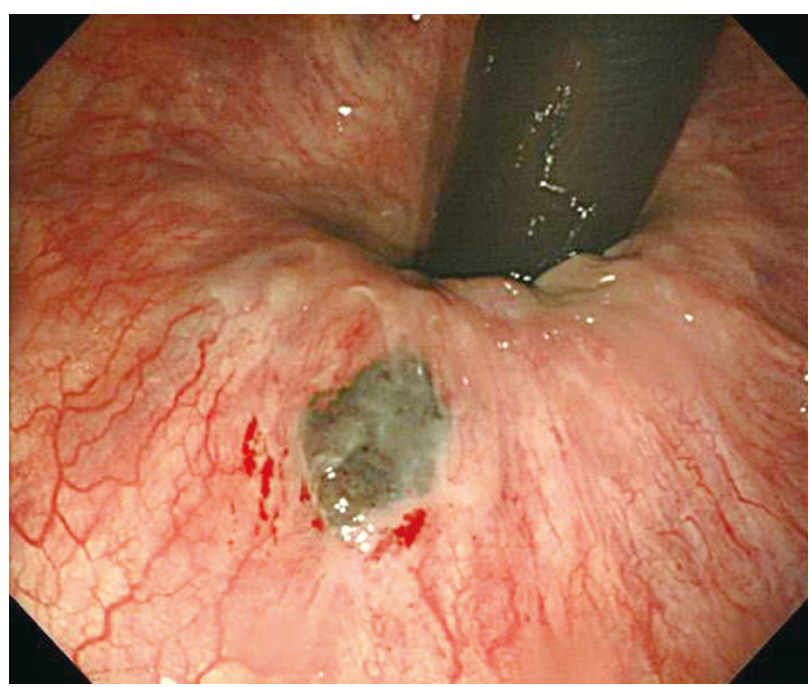

Fig. 1. Endoscopic finding of rectal melanocytic nevus. A $1.0 \times 0.8 \mathrm{~cm}$ hyperpigmented and slightly elevated lesion is visible at the far distal rectum adjacent to the dentate line.

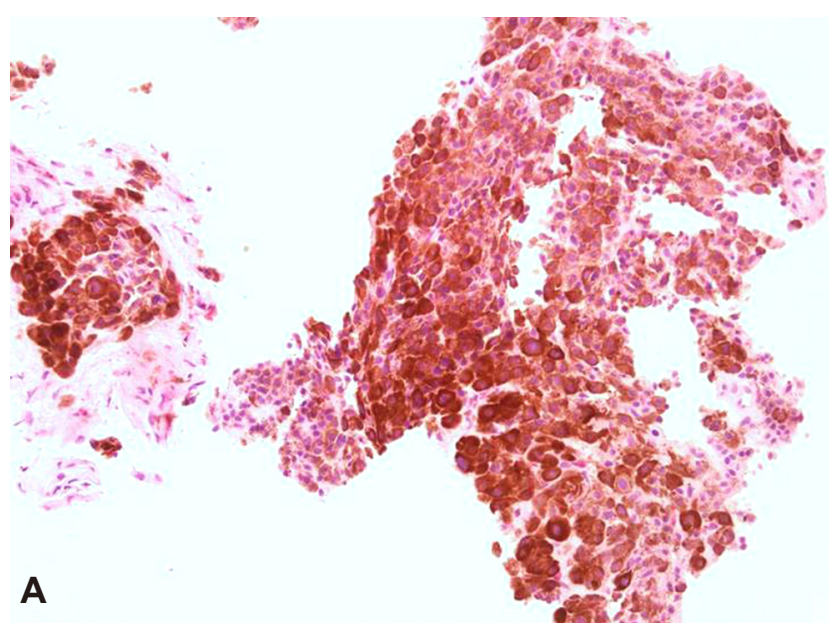
change in size, and under midazolam- and propofol injec-

During the procedure, the patient was stable without major complications. The acquired specimen was fixed in $4 \%$ formalin and sent to the pathology department. The lesion was $0.7 \times 0.7 \mathrm{~cm}$, and hematoxylin-eosin staining and S-100 using immunohistochemical staining revealed many melanocytes and positivity for S-100 stain. Therefore, the resected specimen was finally diagnosed as a melanocytic nevus with no malignant cells (Fig. 4).

\section{DISCUSSION}

Melanocytic nevus has the potential for malignant transformation, and if malignant melanoma is developed on the colon or rectum, severe adverse effects such as diarrhea, rectal bleeding, constipation, and obstruction may occur. ${ }^{3}$ However, in many cases, such side effects may not be clear, making it difficult to diagnose malignant melanoma in the lower gastrointestinal tract. ${ }^{7}$ Up to $1 \%$ of total melanoma cases are the anorectal type, the prognosis of which is known to be generally poor. ${ }^{8,9}$ Considering the potential risk for malignant transformation, if melanocytic nevus is found on the rectum, a biopsy should be taken to confirm the presence of malignant cells. Furthermore, either colonoscopic surveillance or resection should be considered despite a benign pathologic examination. However, there has been no case report of the malignant transformation of rectal melanocytic nevus, and the incidence of malignant transformation of rectal melanocytic nevus remains unknown. Therefore, endoscopic resection using ESD may be preferred if regular surveillance colonoscopy is unavailable or if the patients are younger or want to remove the lesion. To our knowledge, this is the first case in which ESD was used to provide therapeutic resection of rectal melanocytic nevus. However, a treatment consensus should

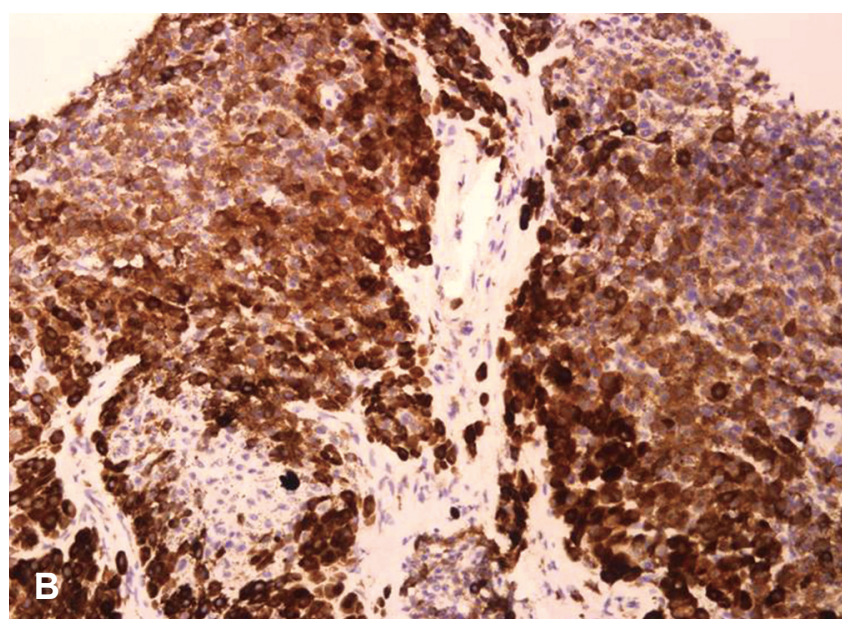

Fig. 2. Histopathological findings of endoscopic biopsy tissue. (A) The sample is composed of many melanocytes and brown pigmentation without malignant cells (H\&E stain, $\times 40)$. (B) The Ki-67 labeling index of the specimen is $<1 \%(\times 200)$. 

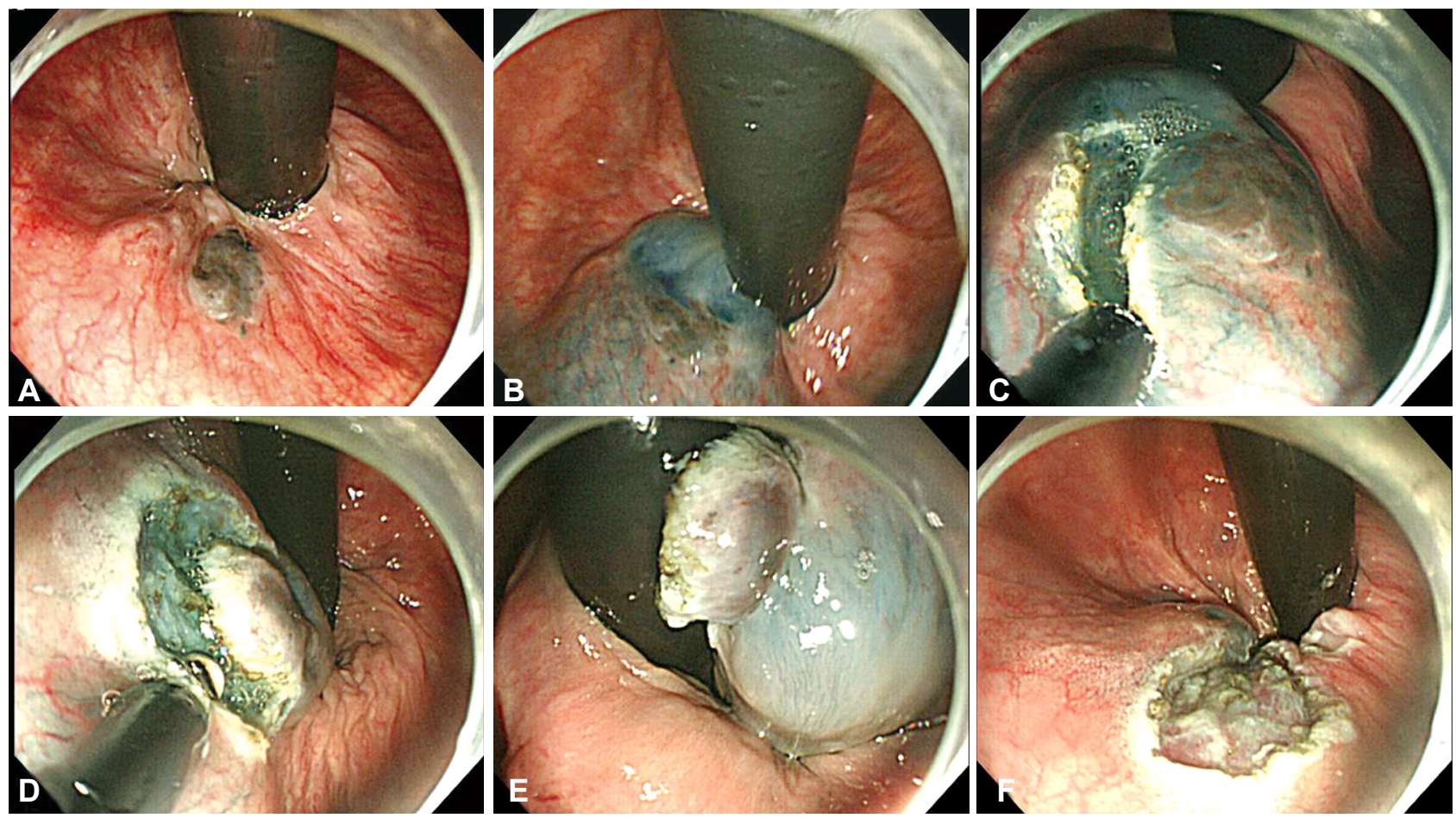

Fig. 3. Endoscopic submucosal dissection of melanocytic nevus of the rectum. (A) A rectal melanocytic nevus is visible on the far-distal rectum. (B) A submucosal injection is performed using epinephrine-indigo carmine-saline solution. (C) A mucosal incision around the lesion is made using a needle knife. (D, E) The submucosal dissection is performed using an insulated-tip knife. (F) The rectal melanocytic nevus is completely removed without major complications.
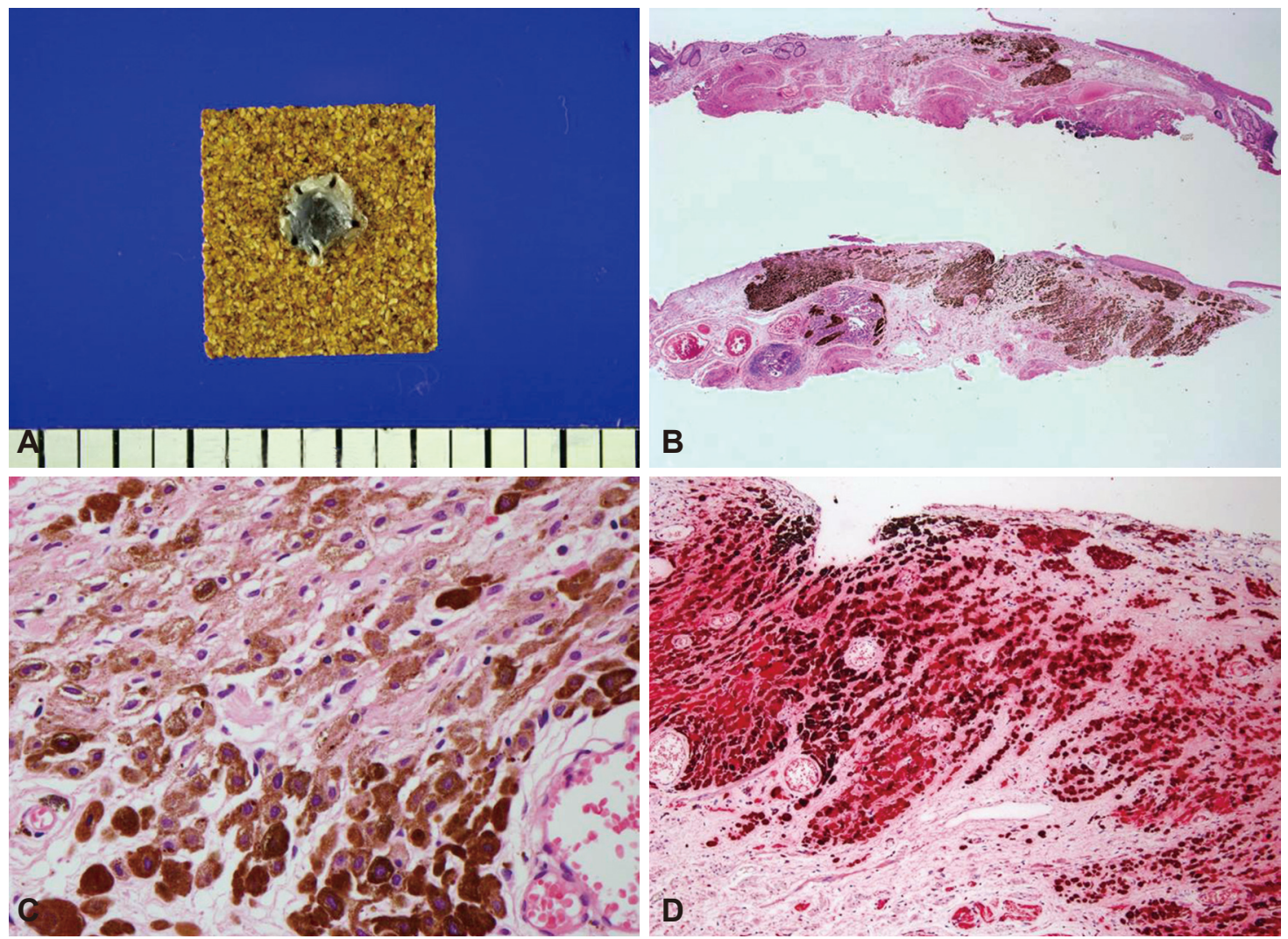

Fig. 4. Histopathological findings of the resected specimen. (A) The gross findings of the specimen indicate that the lesion is $0.7 \times 0.7 \mathrm{~cm}$. (B) The nevus involves the submucosa of the anorectal junction (H\&E stain, $\times 12.5)$. (C) The resected lesion is composed of many melanocytes without malignant cells. Almost all of the cells include brown pigmentation (H\&E stain, $\times 400)$. (D) The lesion is positive for S-100 $(\times 100)$. 
be established by future case reports and long-term follow-up data of endoscopic resection of rectal melanocytic nevus.

In conclusion, rectal melanocytic nevus may have the potential for malignant transformation; therefore, periodic monitoring via colonoscopy should be performed. However, colonoscopic surveillance requires patients to complete a cumbersome bowel preparation process and may increase their anxiety of malignant transformation during the longterm follow-up period. In such cases, removal of the rectal melanocytic nevus can be considered a definite treatment option, and endoscopic procedures including ESD can be safe and feasible alternatives.

\section{Conflicts of Interest}

The authors have no financial conflicts of interest.

\section{REFERENCES}

1. Gilchrest BA, Eller MS, Geller AC, Yaar M. The pathogenesis of melanoma induced by ultraviolet radiation. N Engl J Med 1999;340:1341-1348.

2. Chaudru V, Chompret A, Bressac-de Paillerets B, Spatz A, Avril MF, Demenais $\mathrm{F}$. Influence of genes, nevi, and sun sensitivity on melanoma risk in a family sample unselected by family history and in melanoma-prone families. J Natl Cancer Inst 2004;96:785-795.

3. Purdue MP, From L, Armstrong BK, et al. Etiologic and other factors predicting nevus-associated cutaneous malignant melanoma. Cancer Epidemiol Biomarkers Prev 2005;14:2015-2022.

4. Bataille V, Bishop JA, Sasieni P, et al. Risk of cutaneous melanoma in relation to the numbers, types and sites of naevi: a case-control study. $\mathrm{Br}$ J Cancer 1996;73:1605-1611.

5. Lynch HT, Frichot BC 3rd, Lynch JF. Familial atypical multiple mole-melanoma syndrome. J Med Genet 1978;15:352-356.

6. Harrison SL, MacLennan R, Speare R, Wronski I. Sun exposure and melanocytic naevi in young Australian children. Lancet 1994;344:15291532

7. Patrick RJ, Fenske NA, Messina JL. Primary mucosal melanoma. J Am Acad Dermatol 2007;56:828-834.

8. Khan M, Bucher N, Elhassan A, et al. Primary anorectal melanoma. Case Rep Oncol 2014;7:164-170.

9. Mason JK, Helwig EB. Ano-rectal melanoma. Cancer 1966;19:39-50. 